LAWRENCE LIVERMORE N A T IO N A L LABORATORY

\section{Efficient Web Change Monitoring with Page Digest}

D. J. Buttler, D. Rocco, L. Liu

February 23, 2004

World Wide Web Conference

New York, NY, United States

May 17, 2004 through May 22, 2004 
This document was prepared as an account of work sponsored by an agency of the United States Government. Neither the United States Government nor the University of California nor any of their employees, makes any warranty, express or implied, or assumes any legal liability or responsibility for the accuracy, completeness, or usefulness of any information, apparatus, product, or process disclosed, or represents that its use would not infringe privately owned rights. Reference herein to any specific commercial product, process, or service by trade name, trademark, manufacturer, or otherwise, does not necessarily constitute or imply its endorsement, recommendation, or favoring by the United States Government or the University of California. The views and opinions of authors expressed herein do not necessarily state or reflect those of the United States Government or the University of California, and shall not be used for advertising or product endorsement purposes. 


\section{Efficient Web Change Monitoring with Page Digest}

\author{
David Buttler \\ Center for Applied Scientific \\ Computing \\ Lawrence Livermore National \\ Laboratory \\ Livermore, CA 94551 \\ buttler1@IInl.gov
}

\author{
Daniel Rocco \\ College of Computing \\ Georgia Institute of \\ Technology \\ Atlanta, GA 30332 \\ rockdj@cc.gatech.edu
}

\author{
Ling Liu \\ College of Computing \\ Georgia Institute of \\ Technology \\ Atlanta, GA 30332 \\ lingliu@cc.gatech.edu
}

\begin{abstract}
The Internet and the World Wide Web have enabled a publishing explosion of useful online information, which has produced the unfortunate side effect of information overload: it is increasingly difficult for individuals to keep abreast of fresh information. In this paper we describe an approach for building a system for efficiently monitoring changes to Web documents. This paper has three main contributions. First, we present a coherent framework that captures different characteristics of Web documents. The system uses the Page Digest encoding to provide a comprehensive monitoring system for content, structure, and other interesting properties of Web documents. Second, the Page Digest encoding enables improved performance for individual page monitors through mechanisms such as short-circuit evaluation, linear time algorithms for document and structure similarity, and data size reduction. Finally, we develop a collection of sentinel grouping techniques based on the Page Digest encoding to reduce redundant processing in large-scale monitoring systems by grouping similar monitoring requests together. We examine how effective these techniques are over a wide range of parameters and have seen an order of magnitude speed up over existing Web-based information monitoring systems.
\end{abstract}

\section{INTRODUCTION}

The World Wide Web offers a unique publishing medium that enables information broadcast with few of the traditional barriers to widespread communication. This freedom has fostered growth of online communities that publish information on a near limitless array of topics. Automatic Web change monitoring provides several compelling advantages even for simple scenarios. First, automatic systems remove the burden of monitoring from the user, allowing them to concentrate on other efforts while being assured of receiving timely notification when an interesting change occurs. Second, Web change monitors can track many different sources simultaneously: users can handle more data effectively, making them more productive and increasing the quality of their decisions.

We present an automatic Web change detection system that provides a mechanism for monitoring Web information sources. Our system also offers semantically rich data processing services that provide fine granularity change detection with more expressive power than simple Boolean change tests. This paper describes salient features of our architecture, addressing areas of data management that offer opportunities for optimization. The design provides a framework for flexible and scalable Web change monitoring through the use of: Efficient Data Management using the Page Digest format [2]; Rich Processing Constructs for targeted content and structure; and Grouping popular pages, eliminating redundant computation.

\section{ARCHITECTURE}

Copyright is held by the author/owner(s). WWW2004, May 17-22, 2004, New York, NY USA. ACM xxx.xxx
The general architecture of our Page Digest sentinel system is a third-party change monitoring system handling queries and data on behalf of users, while maintaining independence from the data sources. The core component is the sentinel manager, which installs and removes sentinels, triggers sentinel evaluation at the user specified interval, and intelligently batches queries to maximize processing efficiency over popular targets.

The sentinel manager's central role in our Web change monitoring system mandates efficient processing of sentinels to ensure system scalability. One of the most important optimizations in the sentinel manager is the grouping of related sentinels together to minimize redundant processing and $\mathrm{I} / \mathrm{O}$.

Page Digest Sentinels. An important problem affecting the scalability of any system that interacts with the Web is the processing of standard HTML and XML Web documents. Present Web document formats are redundant and intermix the structure, tag names, attributes, and content. These drawbacks lead us to consider an alternate data encoding that would facilitate fast algorithms and largescale optimizations. The Page Digest Web document encoding [2] increases processing efficiency in our Web document monitoring system by providing direct access to the different semantically interesting characteristics of a Web document while eliminating tag redundancy.

\subsection{Web Document Monitoring}

We now consider the challenges of a Web document monitoring system, focusing on our use of the Page Digest encoding to enhance the system's efficiency. The first issue is that of user interaction, which can be divided into two subproblems: query specification and user notification. The second issue is that of data management, which includes document storage, change evaluation, versioning, and effective use of compute resources. Here we only examine the query specification and data management problems.

Update Semantics. There are two elemental types of changes, and therefore sentinels, with respect to Web documents: content changes and structural changes. Content changes include any change to text and changes to hyperlinks or images. In contrast, structural changes modify the tag structure of the document and alter the relationships between document elements.

We expand the semantic flexibility of these two basic change types along several refinement axes. First, structural changes can be restricted to a particular logical group of structural elements, such as attribute alterations. Second, users will typically not be interested in changes across an entire document; rather, the desire to "hone in" on an area of interest leads to refinement by location. Third, an update may be triggered only if it satisfies some regular 
expression pattern or structure expression. Fourth, an interesting change may be defined in terms of combinations of the two basic change types with any of the refinement modifications present. Finally, a change may be detected over a custom interval, although our implementation restricts this to be a positive integer multiple of a minimum polling interval.

\subsection{Page Digest Sentinel Processing}

The challenge in optimizing a monitoring system is in determining the primary costs and implementing effective schemes to minimize those costs. Those costs can be broadly categorized as network costs, data management costs, and processing costs. Network costs are beyond the control of a third-party system. Data management costs are partially addressed by the Page Digest format; in addition, our system maintains document signatures and sentinels in memory, eliminating a large fraction of local I/O costs. Here we focus on local processing costs incurred during the evaluation of sentinels and describe how we leverage the Page Digest data structure to alleviate the costs.

The implementation of sentinels in our Web change monitoring system deviates from the intuitive notion of a sentinel presented above. Rather than sentinels existing as autonomous agents, our system employs a sentinel manager which is responsible for processing sentinel queries. Since the goal of our system is scalability, our implementation seeks to maximize throughput and may sacrifice individual sentinel latency if necessary. We maximize throughput in two main ways: minimizing redundant network access and minimizing local processing.

Shortcut Techniques. Preprocessing Depending on the type of sentinel and the cost, documents may have signatures created to shortcut processing when no change occurs. Location masking Sentinels may mask out the interesting portion of a document, reducing the computation required for all change detection algorithms.

Content Sentinels. Text change detection operates over the document's content list. The algorithms match user-defined regular expressions or keyword phrases directly over the content container, automatically bypassing other parts of the document. The separation of document components in the Page Digest encoding allows text change detection to operate efficiently over the text without introducing extraneous computational overhead for parsing different document elements during the search.

Structure Sentinels. The Page Digest encoding allows structure sentinels, which ignore the text content of Web documents, to avoid loading the entire document into memory for processing. In many cases, the structure of an HTML document may be small enough to maintain in memory with the sentinel. Structure information can be used to dramatically speed up the execution of generic tree-totree change detection algorithms in the case where there are few or no differences between the document's structure. In addition, the structure information may be used to focus in on specific features, such as links for highly-efficient scanning.

\subsection{Multiple Sentinel Processing}

By combining related requests, we are able to minimize network costs and reduce processing costs with only a modest processing overhead for group maintenance. Sentinel grouping allows the system to execute a single document fetch for all sentinels over a certain document. This optimization assumes that all sentinels over the document expect updates at the same interval. For groups where this is not the case, the sentinel grouping process temporarily re- moves sentinels that are not due for notification from the group.

Coupled with the Page Digest encoding, grouping allows the system to perform change detection only once for each group, efficiently reusing the change information for individual sentinels. Grouping also eliminates redundant processing by executing more general sentinels in the group before more specific ones.

Group processing begins when the sentinel manager is triggered to check for changes that affect the group. The sentinel manager retrieves all sentinels installed on the current URL and selects the active set for this firing interval. The new version is retrieved from its source, hashed to a signature, and if changed the previous version is loaded from disk.

The sentinel manager computes an annotation array, marking what has changed, then examines the locations in the annotation array specified in each sentinel's location mask. Comparison begins with the sentinels in the minimal covering subset, which is the smallest subgroup of sentinels that covers every location of interest to the group for each particular change type. If none of the sentinels in this subgroup detects a change, no further processing is needed.

\subsection{Experimental Summary}

We performed several experiments to measure the system against other systems and techniques, and to demonstrate the power of our optimizations, four of which we mention here. Our first experiment compared the overall performance of the sentinel processing system to the WebCQ system [1]. Each of the novel characteristics in the Page Digest sentinel system addresses performance bottlenecks from this system, allowing us to achieve between one and two orders of magnitude speedup in processing time. Our second experiment examined sentinels that were grouped in a Zipf-like distribution over common Web pages. By combining processing between similar monitoring requests we were able to improve the execution time by $50-70 \%$. The third experiment examined the cost of grouping large numbers of sentinels, showing that the cost is amortized over the size of the group, costing less than loading a document into memory, and approximately the cost of computing an MD5 signature for the document. The final experiment demonstrated the performance increase of using the Page Digest format over using the more popular DOM tree, at less than half the cost for the same sentinel type.

\section{CONCLUSION}

We have presented a new mechanism for detecting changes to HTML and XML documents based on the Page Digest encoding, focusing on providing standard change detection mechanisms, operators for more advanced change detection operators, techniques to monitor different aspects of a page, including content, tag types, attributes, and structure, and, finally, a set of unique optimization techniques based on the Page Digest encoding that dramatically improves the performance of the system.

Acknowledgments: This work was partially performed under the auspices of the U.S. Department of Energy by University of California Lawrence Livermore National Laboratory under contract No. W-7405-ENG-48. UCRL-CONF-202469.

\section{REFERENCES}

[1] L. Liu, C. Pu, and W. Tang. WebCQ: Detecting and Delivering Information Changes on the Web. Proceedings of the International Conference on Information and Knowledge Management, November 2000.

[2] D. Rocco, D. Buttler, and L. Liu. Page digest for large-scale web services. In Proceedings of the IEEE Conference on Electronic Commerce, 2003. 